\title{
Requirements Engineering for the Ageing Population: a Teaching Perspective
}

\author{
Amélie Lachapelle-Dagenais*, Sébastien Mosser*, Anne-Marie Pinna-Déry ${ }^{\dagger}$ and Mireille Blay-Fornarino ${ }^{\dagger}$ \\ ${ }^{*}$ Université du Québec à Montréal (UQAM), Montréal, Canada. \\ email: \{lastname.firstname\}@uqam.ca \\ †Université Côte d'Azur, I3S, CNRS, Sophia Antipolis, France. \\ email: \{firstname.LASTNAME\}@univ-cotedazur.fr
}

\begin{abstract}
Elders occupy an ample space among the potential end-users of the pieces of software we are designing. This situation is only increasing, and even the pessimistic projections forecast global ageing in the World population. In this context, it is our duty as software engineers to design and implement software adapted to elders. However, this particular problem is too often bypassed in the classical software engineering curriculums. In this paper, we report about a set of courses dedicated to requirements engineering for the ageing population, currently implemented at Université Côte d'Azur (UCA, France) and Université du Québec à Montréal (UQAM, Canada). We identify challenges related to the ageing population that can be addressed in a course and report on how such courses are implemented in both institutions. The work reported in this paper is validated through an in-depth case study at UCA, based on 18 years of experience in the teaching of requirement engineering for specific populations.
\end{abstract}

\section{INTRODUCTION}

As an immediate consequence of the demographic transition model [1], the world population is facing a crisis concerning its elders. For example, in 2016, the percentage of Canadian population [2] in the $65+$ age rank was superior to $15 \%$, representing $5.8 \mathrm{M}$ people (TAB Ia). Depending on the growth projection model used, this number is expected to increase to $\sim 25 \%$ (TAB $\mathrm{Ib}$ ), representing a number of citizens estimated between $9.8 \mathrm{M}$ (low-growth hypothesis) and $10.8 \mathrm{M}$ (highgrowth hypothesis) people [3]. A more recent census makes a distinction between $65+$ and $80+$ categories, with 50 -years projections [4]. If the Canadian population in the $80+$ category was only $4.3 \%$ in 2018 , it is expected to represent up to $12.3 \%$ by 2068 . Even if the crisis is more visible in developed countries, the current situation is a worldwide challenge affecting the global health of the population. According to these projections, the senior dependency ratio (i.e., percentage of the population aged 65 and over per hundred persons aged 15 to 64 .) is expected to be $29.8 \%$ at the World level (median age: 38.2 years), but up to $49.6 \%$ in more developed regions ${ }^{1}$, with a median age of 46.7. In Japan, the senior dependency ratio is expected to reach $75.9 \%$ (median age: 55.1 years).

In such a context, several initiatives arise to train the next generation of software developers. For example, in Canada,

\footnotetext{
${ }^{1}$ According to Statistics Canada methodology, more developed regions comprise Europe, Northern America, Australia/New Zealand and Japan.
}

TABLE I: $65+$ citizens in Canada [2]

\begin{tabular}{c|ccccc} 
Year & 1976 & 1986 & 1996 & 2006 & 2016 \\
\hline Male & $7.6 \%$ & $9.1 \%$ & $10.5 \%$ & $12.2 \%$ & $15.6 \%$ \\
Female & $9.8 \%$ & $12.2 \%$ & $13.9 \%$ & $15.2 \%$ & $18.1 \%$
\end{tabular}

(a) Percentage of citizens from 1976 to 2016

\begin{tabular}{c|ccc} 
Year & Low-growth & Med-growth & High-growth \\
\hline Male & $24.7 \%$ & $23.5 \%$ & $22.7 \%$ \\
Female & $26.8 \%$ & $25.4 \%$ & $24.6 \%$
\end{tabular}

(b) Estimated percentage in 2036 (three growth models)

the AGE-WELL ${ }^{2}$ nationwide network of centres of excellence regroups 45 universities, 400 industrial partners and 200 researchers working together in the context of "technology and ageing". In addition to the research and product incubation areas, the network dedicates resources to support training and has trained more than 760 students since 2015 [5]. More recently, the Natural Sciences and Engineering Research Council of Canada funded for $1.65 \mathrm{M} \$$ a training program entitled SMAP (Smart Mobility for Aging Population ${ }^{3}$ ). In Europe, to the best of the author's knowledge, there is no educational network dedicated to software engineering for the ageing population. At the level of universities, initiatives exist to support educational programs related to "eHealth" in general, but, again, nothing particular to the ageing population.

Even if we can observe a recent trend in teaching initiatives related to Information and Communication Technologies related to eHealth in general and more particularly the ageing population, it is far from typical for universities to include dedicated courses in their programs: notions required to address such population are at best taught to the students as isolated silos, and at worst ignored. Considering the current situation, we defend that it is not reasonable for students in computer science and software engineering not to address this population. It goes again equity, diversity and inclusion criteria. In this context, Université Côte d'Azur (UCA) pioneered in 2003 the inclusion of a project-based course dedicated to societal impact (B.Eng. and M.Eng. programs). The idea was to put students in charge of completing a software development project, emphasizing requirement engineering and adaptation to particular needs. Initially focused on visually-impaired K12

\footnotetext{
${ }^{2}$ https://agewell-nce.ca/about-age-well

${ }^{3} \mathrm{https}: / /$ smap.mcmaster.ca/
} 
children $^{4}$ as end-users, the engineering students were guided in the implementation of serious games dedicated to such population. The scope then evolved to interact with patients who have Alzheimer's disease [6], in collaboration with a local day center and respite halt. Since 2017 UCA and Université $d u$ Québec à Montréal (UQAM, thanks to joint funding received from the French and Quebec's governments), developed a set of shared courses specifically dedicated to the ageing population.

This paper reports on developing such a course from a requirement engineering point of view. We identify in SEC. II the challenges faced by the students in this context. Then, we describe in SEC. III the problem-based methodology followed in the course to support the students from the requirements engineering phase to the implementation and validation ones. In SEC. IV we propose an in-depth case study to explain how UCA implemented the course earlier this year. Finally, we will discuss some lessons learned for the teaching of requirements engineering in the particular context of this course (SEC. V), before concluding the paper in SEC. VI.

\section{Challenges in Adapting Software to Elders}

Developing a software project dedicated to elders is challenging by itself. To address this from a teaching point of view, one needs to take into account two different kinds of challenges: $(i)$ the ones related to the domain, and (ii) the ones related to what can be taught during a session. As an educator, it raises the following question: "What can I cover during my course, and to which depth can I dive?". This section proposes a curated list of themes that can be considered as teaching support at the undergraduate level, based on our experience and the requirements engineering perspective. We distinguish between what comes from the domain and what comes from the teaching environment for each theme. We voluntarily addressed themes related to impairments related to ageing, as they are, from our experience, the most difficult to manage with students in terms of requirements.

\section{A. Unavailability of end-users}

a) Domain-knowledge: In the context of an ageing population, it is often not possible to have direct access to the stakeholder with the proper knowledge. For example, consider a health-monitor application that advises the elders concerning their mobility. When considering a persona suffering from dementia (and then adapt the way the advice is given to take care of the cognitive loss associated with this pathology), it is challenging to conduct interviews and capture relevant requirements. Instead, the requirement engineer has to use translated requirements obtained through the interview of caregivers (family or professional) and medical staff. A caregiver will identify which advice needs to be recommended, and the

\footnotetext{
${ }^{4}$ DEVINT, DÉficients VIsuels \& Nouvelles Technologies - Visual Impairment and New Technologies. The pedagogical approach implemented in this course was ranked first in a national competition related to teaching for impaired children, and was then presented at the World Usability Day in 2007.
}

medical staff can explain how such advice can be communicated efficiently while taking the pathology into account. Then it is up to the requirement engineer to reconcile partial pieces of information into a complete set of requirements and to find a way to validate this reconciliation with the stakeholders.

Another challenge related to this situation is faced when validating the developed products. Classical agile development relies on product demonstration that involves the end-users to collect feedback. However, in the previously described context, elders are not always capable of verbalizing their feedback. Thus, the validation cannot be performed directly and must, again, rely on a transitive validation: the product is given to the end-user for demonstration purposes, but the feedback is collected through the caregivers or medical staff who observed end-users behaviour. Going back to the previous example, a person with dementia might not tell why he or she made a particular action, such as moving from a room to another one. However, an attentive observer, who knows the person's typical behaviour, can identify that this action was triggered by a piece of advice sent by the app, validating that the way the advice is given is appropriate.

b) Teaching: Teaching requirements engineering is already hard enough when you have direct access to your stakeholders. The elicitation of these requirements is usually done through interviews and focus-group with representative people. The challenge here is to teach students to gather requirements from other stakeholders and translate these pieces of information into precise requirements. When evaluating a solution, students will have to observe more than ask for feedback. Therefore, instructors must familiarize them with confirmation biases in this context (e.g., ignoring observations that do not fit their assumptions).

\section{B. Visual Impairment}

a) Domain-knowledge: The spectrum associated with visual impairments is vast. However, it is possible to focus on several troubles that are problematic for elders. If some troubles can be found in the general population (e.g., myopia, astigmatisms), others have an increased prevalence in the ageing population, like diabetic retinopathy, Age-Related Macular Degeneration (ARMD), or cataract. For example, ARMD is a common ${ }^{5}$ degenerative disease that blurs the center of the vision and distorts the peripheral view. In such a context, increasing the font size is a wrong adaptation for elders, as it will increase the part of the text that is blurred. In this case, it is better to use the center of the screen as little as possible and display the text using the periphery of the screen, with a font that supports distortion.

Another interesting requirement here is related to the degenerative and non-curable characteristics of these diseases. It is not possible to adopt a silver bullet adaptation, as it is necessary to adapt the system to the current state of the user. Considering ARMD again, an elder at the first stage of the

\footnotetext{
${ }^{5}$ The number of people living in the USA suffering from ARMD is almost equivalent to the number of people living in Québec ( 8 million).
} 
disease will only suffer from mild discomfort initially and will find it odd to have all the information pushed to the screen periphery for no apparent reasons. So the graphical user interface has to follow the stage of the elder while using the application, leading to complete blindness in the end. At this stage, it is not even possible to use the initial application, and a vocal version must be used.

b) Teaching: From a requirement engineering teaching point of view, it is interesting here to deconstruct the a priori ideas that students have about visual impairments related to ageing. Classically, they believe at first glance that increasing the font size is sufficient to provide an adapted version for elders, relying on the ageist cliche that "elders need glasses". It is interesting to illustrate these visual impairments by relying on simulators ${ }^{6}$ to demonstrate that students' initial ideas are unsuitable for designing an application that fits elders' needs.

\section{Motor Impairment}

a) Domain-knowledge: Ageing comes with a large spectrum of impact on the autonomy of the elders. It is possible to focus on what characterizes ageing concerning motor skills, i.e., the progressive loss of fine-grained mobility. Another dimension that can be explored in this context is related to Artificial Intelligence (AI), through the prediction of trends associated with motor skills' losses. In the end, it becomes possible to map the work done with simple sensors (e.g., using smartphones or tablets accelerometers [7]) to classical living labs, which also uses sensors to monitor elders activities.

b) Teaching: Taking care of motor impairments can be achieved in a curative way by adapting the graphical interface to avoid small buttons or tiny elements. This is particularly true when using a mobile application as a target, considering that the sizes of smartphone or tablet screens can be challenging for elders. These losses can also be considered in a preventive way by measuring them and monitor their evolution. For example, considering a mobile application again, it is possible to use the accelerometer sensor to measure the handshaking of an elder using the application while he or she is using it. Being able to trace and record the precision of an elder when he or she is using the application is essential to evaluate the graphical user interface, identifying misclicks, for example. Available datasets [8] of smartphones' movements can be used to train AI-based classifiers and explore how to classify and predict human behaviour based on sensor data. Thus, it is possible to demonstrate how to train such a classifier using one of the available datasets. When the students understand how such prediction approaches work and what a suitable dataset is in this context, they can design suitable data collection mechanisms.

\section{Cognitive Impairment}

a) Domain-knowledge: Like for motor impairments, ageing has a broad spectrum of effects that impact elders' cognitive skills, from small memory losses to Alzheimer's

\footnotetext{
${ }^{6}$ e.g., http://www.inclusivedesigntoolkit.com
}

disease, including any degrees of dementia. These effects can be addressed according to two different approaches: preventive or palliative. For prevention purposes, the implementation of serious games in link with psychologists is an excellent way to support elders [9]. The games can be developed generically (e.g., memory games) or designed to focus on specific individuals. For example, one can design a quiz or memory game where the data used to feed the game are collected from the elder's family. Thus, he or she will be playing using his or her memories, keeping them alive. The palliative approach consists in adapting the application mechanics to elders who have reached intense stages of such degeneration. For example, elders who have dementia usually do not accept the fact that they are making mistakes. As a consequence, where a given piece of software would have presented an error message (e.g., red text stating "this field is missing" in a given form), an adapted version will have to re-ask the elder for input, in a different way (e.g., presenting a new form asking for the same information with an alternative label).

b) Teaching: The main challenge here is to support the collection of requirements, as interacting with people suffering from these troubles can be very destabilizing for students. The opposite side of the coin is the triggering of difficulties in adequately evaluating elders as end-users. This can be mitigated by replacing elders with caregivers or instructors familiar with these troubles or directly by medical staff.

\section{E. Composition of impairments}

a) Domain-knowledge: None of the previously described dimensions are specific to ageing. For example, dementia was previously identified as senility, or senile dementia. However, it is now accepted that this kind of health issue is not a normal effect of ageing. Ageing is a composition of minor impairments that change the way an elder can interact with technology. Thus, adapting software to elders is not the same as adapting software to blind people or Alzheimer's patients. It is about considering a potential loss of sight, coupled to a potential loss of cognitive reactivity, coupled to...

b) Teaching: The critical challenge here is finding the right trade-off between being too superficial during the semester to cover as many situations as possible and diving into a given affliction, which would not represent elders' requirements. This risk can be mitigated by conducting interviews with elders as part of the course, for example, thanks to a partnership with a local retirement residence. Caregivers are also important stakeholders, as their vision of the needed requirements aligns well with one of the elders. It might be more simple for students to interact with caregivers.

\section{Methodology: A Problem-BASEd ApproACH}

To address these challenges we developed a course setup that was successfully implemented in three different undergraduate contexts: (i) B.Eng. at Polytech Sophia (UCA), (ii) Licence professionelle at IUT Nice - Côte d'Azur (UCA) and (iii) B.Sc. at UQAM. 
- Persona: Dominic

- Job: Retired (formerly: Air Canada pilot)

- Age: 83 years old

- Technological skills: Average

Dominic has been living in an elders' residence for two years now. He is very sociable and has a wide range of interests, and has quickly made friends. He is frequently visited by his son Marc, to whom he is very close. Over the past few months, Dominic has noticed that his memory is not as sharp as it used to be. He seems to forget more and more things. This is very confusing and makes him a little irritable. He has reported this to the institution's medical staff and is being followed by Daria, a psychologist. No diagnosis has been made yet. He has always been interested in technology, but he has felt overwhelmed for the last 10-15 years: everything is changing so fast. He hopes to eventually tame Bob, the name he gives to his smartphone.

Fig. 1: Description of a persona and his interactions

The course used a problem-based approach. Students are exposed to a given problem thanks to introductory lectures and then propose solutions to tackle the presented problems. The proposed solutions are then evaluated by the staff (a combination of instructors and end-users), and feedback for improvement is given to the students.

\section{A. Role of personas}

In the context of problem-based learning, the role of personas [10] is essential for requirements gathering. A persona is a fictive character that will have to interact with the designed project as an end-user. Asking for detailed descriptions (Fig. 1) helps the students to avoid mistakes by asking themselves the question: "If I was $<\mathrm{X}>$, would I perform this action? Would I seek for this benefit from the app?". During the first weeks of the course (for around $\sim 25 \%$ of the course), instructors work with students to characterize the right personas. In our context, it can be an elder, a caregiver, family members, or medical staff. The personas definition is reviewed by experimented staff, e.g., real stakeholders or instructors with an in-depth knowledge of such situations.

\section{B. Requirements as user stories backlogs}

Among the different approaches of expressing requirements, we chose to use User Stories. Stories are expressed in natural language, using the classical template "As $<$ persona $>, I$ want to $<$ action $>$ so that $<$ benefit $>$ ". Using only stories to represent requirements is controversial [11], as, by definition, a user story describes a feature but does not provide any details about how to implement it (and thus not being an agile equivalent of a requirements specification). However, typical RE courses often cover agility [12].

Moreover, by pushing the students to describe features related to personas and identify the added benefit for a persona to perform such an action (Fig. 2), it is the best way we have found to make students focus on the difficulty of adapting
- As Dominic, I want to activate the collaborative mode so that I can play with my son when he visits me.

- As Dominic, I want to have access to personalized quizzes based on my memories so that I can exercise my memory skills.

- As Daria, I want to monitor the residents' responses so that I can identify early signs of cognitive impairment.

- As Marc, I want to share pictures and stories about my father with the residence staff so that they will be able to design personalized quizzes for him.

Fig. 2: Excerpt of a backlog for a quiz-like serious game

software to elders. It allows students to be creative in how they solve the problems they are working on: they are entirely free in the kind of stories they are defining, as soon as they can defend to the instructors that realizing this story in their product will provide a benefit to the associated persona.

The safety net created by investing time in the persona's definition is here essential. Even if user stories are not the most formal way of expressing requirements, their flexibility is an essential trade-off for the design of the course. Focusing on the benefits associated with each action forces students to wear their personas' shoes and precisely identify how the thing they are designing will be helpful. The fact that they are defining their requirements as stories backlog also provides flexibility in terms of planning. It is possible to describe the first problem to the students (e.g., "how to stimulate elder's memory by leveraging their close family?"), let them work for a couple of weeks, and then introduce a new problem on top of the previous one (e.g., "how to monitor fine motor skills losses?").

\section{Evaluation of students results by end-users}

The evaluation of the products designed by the students is essential, as they are designing applications for stakeholders they cannot impersonate easily (because of the generation gap). To support such end-user evaluation, we usually invite practitioners from our network and elders to visit the university and act as end-users. Students demonstrate the product using classical Human-Computer Interaction (HCI) evaluation methods by preparing evaluation scenarios, monitoring (e.g., filming) the user while he or she is following the scenario, taking notes and collecting feedback when possible. We usually organize two to three evaluation sessions during the semester, so students' teams can integrate feedback and iteratively improve their product. We evaluate how they collect feedback and their capacity to integrate such feedback in their product backlog to prepare the next development iteration.

\section{Logistics}

When building this requirements engineer course, we decided to take advantage of the specificity of each research environment, providing a standard kernel that was then adapted to each teaching location. For the first two years, the kernel 
used the same topic as the running project. It allowed the instructors' team to build a strong knowledge of the subject and to design the teaching material ceteris paribus. At UQAM, we decided to focus the course on artificial intelligence and mobile programming, to fill some gaps in the elective course portfolio. UCA, on the other end, focused on the development of teaching material related to web programming. In both cases, the material designed to support requirements engineering, ageing population needs and software evaluation was reused in both institutions.

a) Licence professionelle at UCA: Two co-op cohorts are participating in this course, which lasts six weeks divided into two sequences. The first two weeks aim at giving the basics of project management (e.g., requirements engineering, kanban). For the following four weeks, instructors emphasized acceptance testing. The course uses connected devices as experimental material to interact with the elders, and taking into account the effects of ageing is mandatory at the requirements collection and implementation levels.

b) B.Eng. \& M.Eng. at UCA: The School of Engineering implements two courses taking into account ageing: one at the B.Eng. level (see SEC. IV), and one at the M.Eng. level. The B.Eng. course lasts 12 weeks, divided into three sequences of four weeks. The first period consists mainly of a tutorial focused on web technologies (e.g., Angular, NodeJs) and requirements collection, user-centred design and mock-ups. At the end of this sequence, students deliver a set of mock-ups to be evaluated by external partners (e.g., caregivers, elders) and a web application to demonstrate their technical skills. The second sequence focuses on ageing-related requirements' implementation. A demonstration concludes this sequence in front of all the instructors. The last sequence is dedicated to including users' feedback (from instructors and peer reviews between the teams) in the application to increase the acceptance level. It ends with a demonstration held in front of all the instructors and external partners.

The M.Eng. course is dedicated to HCI and leverages an interactive table that has the advantage to facilitate collaborative workshops carried out in respite halls [6]. The development is done with a user-centred approach and agile management, with close collaboration with the external partners (three times out of eight weeks, for two teams).

c) B.Sc. at UQAM: The course is implemented as four sequences of four weeks each. Each sequence starts with a three-hour lecture session that gives all the necessary theoretical material for the sequence. Then, students' teams have up to two weekly appointments with an instructor (selected TAs or prof) to describe how the project is going and receive feedback from the instructors. The first sequence is dedicated to personas definitions, and the second sequence supports mobile application development. As a consequence, students can demonstrate for mid-terms a mobile application dedicated to elders. The third sequence is related to AI and leverages the sensors from mobile devices to implement behavioural recognition. Finally, the last sequence is an open one, where the students negotiate what they want to do with the instructors (e.g., stabilize what they already have, explore a new direction in terms of adaptation)

d) Impact of the COVID-19 crisis: The COVID-19 crisis impacted the end-user evaluation, considering that elders were the class of population that needed to be the most protected from the disease. As a consequence, we had to cancel these end-user evaluations in 2020 and 2021. We replaced the evaluation with demonstration videos, where students delivered screencasts of their products. The videos were evaluated by the instructors, as well as external practitioners, when possible. We decided not to include elders in the evaluation process, as it is complicated to evaluate an app-based only on a video if you are not comfortable with technology.

\section{CAse study: a Session at UCA}

\section{A. Theme: a Quiz game}

Students have to develop a quiz game grouped as teams of four people. We chose this theme as its business logic is simple enough to focus on the elders' adaptation. The game targets elders who live in specialized institutions (for dependent people) or who use the respite service provided by such institutions during the day. It is possible thanks to a partnership with the Centre d'Accueil de Jour in the city of Biot, a local respite hall.

Each group defines the scope of its project by choosing the impairment(s) they will target to adapt the quiz for elders (among visual, psychological, and motor). They also characterize the different users of their application (e.g., caregivers, family, seniors), and the different actions they can perform (e.g., managing the quizzes, playing, monitoring results, configuring the platform). Communication with users and/or their representatives is central to the success of such projects. Therefore, listening and taking into account needs is essential. Also, each group is asked to prioritize the adaptation to elders before any other functional requirements (i.e., the viability of their minimal and viable product is defined by the way they have adapted the product to elders).

From the student point of view, the challenge is threefold: (i) developing an application from scratch using a new environment (web programming), (ii) becoming familiar with the problems of the ageing population and (iii) taking into account various stakeholders, with different points of view on the very same application.

From the instructor point of view, the challenge is also threefold: (i) provide enough course material to allow the students to create a web-based quiz application easily, (ii) transmit enough information about the different impairments so that students can identify the ones they want to support, and (iii) explain user-centred design [13] to let the students evolve inside the scope of the personas they have identified.

\section{B. Impairments targeted by the teams}

During Winter 2021, the cohort at UCA consisted of 76 students grouped into 19 projects. The instructor staff comprised one professor and two "practitioners" (recent alumni 
who stay involved in the department, acting as an intermediate role between TA and lecturer based on their experience).

Fig. 3a shows the kind of impairments addressed by the teams. It can be seen that the students mainly choose to support requirements related to cognitive and visual impairments. This was influenced by the information provided to the students (e.g., W3C standards for web accessibility, stakeholders from the respite centre) at the beginning of the course. Teams that have made adaptations for multiple impairments have chosen a primary focus area and then added another simple adaptation to make it more inclusive concerning elders' requirements, e.g., combining a font magnification function with cognitive adaptations makes reading easier for elders who also start to encounter cognitive losses.

The teams explored several ways to deal with cognitive impairment (Fig. 3b). For example, they proposed simplifying the information displayed to players identified as cognitively declining, repeating information to preserve active memory as long as possible, and introducing positive incentives to maintain the elders' attention and motivation. They also identify the need for caregivers to monitor the behaviour of the elders, with detailed information that can be used in medical followup appointments.

For visual adaptations (Fig. 3c), the teams focused on the enlargement of the characters' font size and highly contrasted interfaces, as advised by the W3C [14]. Fewer groups considered age-specific adaptations such as ARMD and glaucoma. They also offer solutions for colour-blindness or eye fatigue that are not necessarily related to ageing. The combination of age-specific visual disturbances with younger-appearing disturbances has not been studied during this semester.

For motor adaptations, teams focused on setting up help mechanisms for entering information (i.e., voice command, delay between two clicks, confirmation after a click, and enlargement of the buttons). Like for the cognitive ones, they have also identified the need to monitor the behaviour of the elder while playing, to collect metadata (e.g., recording mouse clicks) that can be used to monitor the health of the player.

All the adaptations were identified by the teams, according to the problem-based approach followed by the course. The instructors provided feedback to advise the teams when they were taking wrong directions or collecting requirements in a bad way. The cognitive adaptations were evaluated by the medical staff from the Centre d'Accueil de Jour, using as reference baseline elders who have Alzheimer's disease.

More details on the solutions proposed by the teams are given in the following section. For each impairment, we highlight the strengths and weaknesses of the proposed solutions. These results shows their understanding of RE.

\section{Results obtained}

Out of the 19 teams involved in the course, only one failed the course. However, results vary significantly from team to team. We classified the adaptations to elder's requirements (for grading purposes) according to two dimensions: technical difficulty and business value (according to caregivers' experience). Tab. II summarize the adaptations implemented by the teams. The remainder of this section discusses how the adaptations identified by the students adequately address elders' requirements.

a) With Respect to Visual impairment: The teams who worked on visual impairments used as input the information covered by the course material. They were not provided with any means to evaluate the validity of their application.

Several teams focused on ARMD, as a relatively common and effective way to compensate for ARMD is to use peripheral vision. Thus, they adapted the application by setting all the elements in the screen's periphery. This kind of adaptation would help somebody with a mild macular degeneration (Fig. 4a) but not necessarily with a severe one: in severe cases, the dark spot will grow too large and cover part of the text, in addition to the blurriness induced by the ailment. This simple adaptation also assumes that the center of the screen is the sight focus. If the eyes move to another part of the screen, then the interface is not usable anymore. A team addressed glaucoma with some success, centring everything in the center of the screen (Fig. 4b). They also identified the feature interaction that exists between this adaptation and the previous one for ARMD. They ensured that both adaptations are mutually exclusive in their configuration tool and cannot be used simultaneously for the same elder.

More generally, we witnessed several teams who realized too late that "perfect is the enemy of good". By letting opened too many choices (e.g., font size, colours, contrast levels), it was easy to configure versions of the quizzes that were unusable. Students learned here the hard way how requirements collection is essential in this context. Even if it is technically possible to do whatever they wanted, the best implementations are the ones offering a limited set of wellthought choices designed to fulfill end-users needs, not the ones that let every option open. This is obviously true for any application, but working with the ageing population made it easy to demonstrate.

All the visual adaptations were evaluated thanks to a simulation tool $^{7}$, and feedback was provided to the teams. We made this pedagogical choice to push the students to look for this kind of tool by themselves, but this assumption was a failure in the end. However, providing a tool since the beginning might be counter-productive, as the students might use the tool as an objective and will not focus on understanding how to fulfill the elder's requirements (e.g., trying to trick the simulator instead of understanding the underlying problem). A trade-off would be to provide the tools after a few weeks of development to confront their vision of the solution to something closer to reality.

b) With Respect to Motor impairment: Out of 19 teams, only four addressed motor impairments for elders. All those teams had good adaptations ideas. An example of a suitable motor adaptation is to record the mouse activity to monitor

\footnotetext{
${ }^{7} \mathrm{http}: / /$ www.inclusivedesigntoolkit.com/simsoftware/simsoftware.html
} 


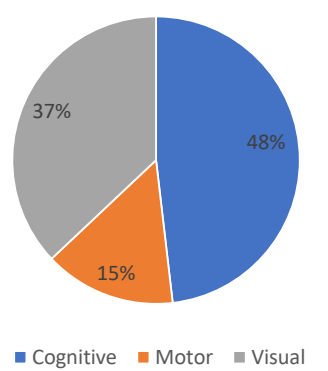

(a) Types of adaptation

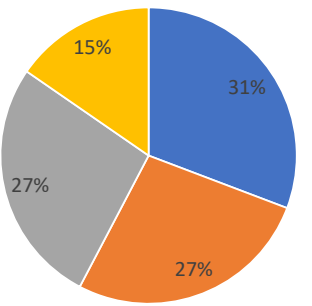

- Simplification $=$ Repetition $\approx$ Support $=$ Statistics

(b) Cognitive adaptations

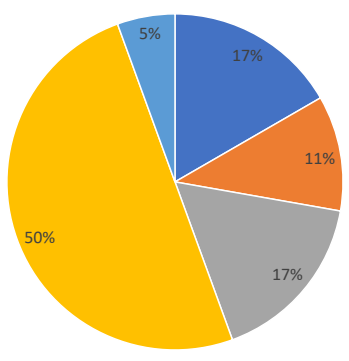

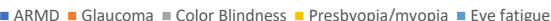

(c) Visual adaptations

Fig. 3: Adaptations targeted by UCA students (Winter 21)

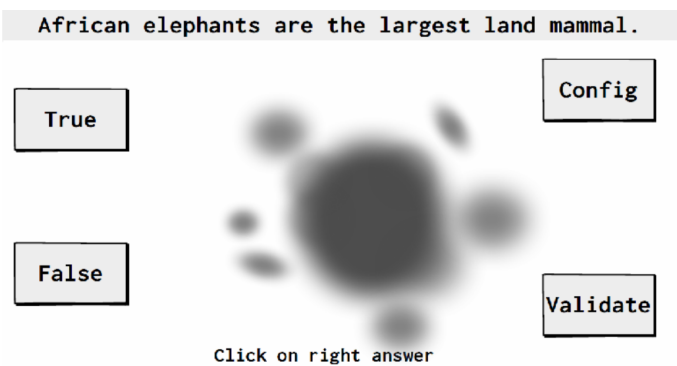

(a) Answering a question, with mild ARMD

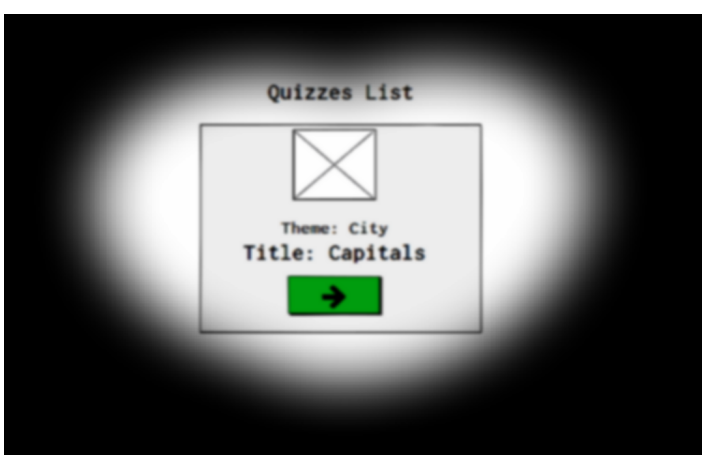

(b) Selecting a quiz, with medium glaucoma

Fig. 4: Visual adaptations evaluated with a simulation tool

elders' behaviour (Fig. 5). A caregiver can compare different recordings throughout time and follow users' trends.

Another remarkable adaptation was to modify the game logic and asking for confirmation when selecting an answer. As an involuntary click can cause frustration and even ruin the user experience for a user, they have proposed additional confirmation screens. This was coupled to additional configuration parameters to slow down the double-click sensitivity for elders with hands shaking (e.g., Parkinson, motor spasms).

This family of impairments allowed instructors to focus on consistency when taking into account such requirements. For example, two teams decided to enlarge buttons to help users, a

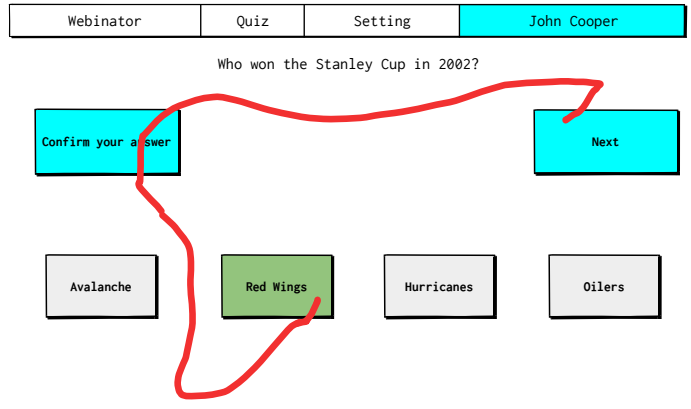

Total number of clicks: 3

Fig. 5: Recording mouse activity

relatively straightforward adaptation for declining motor skills. However, these adaptations needed to be made consistently and are useless if the home screen is not adapted or if the button to go to the next question is still regular.

The teams that focused on motor adaptations delivered applications with fewer features to compensate for the difficulty of implementation. The challenge raised by motor adaptation can partially account for the low number of teams willing to face it. Taking a step back, equipping students with better tools at the beginning (e.g., studies, examples, physiotherapists) could have made the difference.

c) With Respect to Cognitive impairment: Nine teams addressed cognitive impairments during the semester. Considering the history of collaboration between the instructors' research group and Alzheimer's patients and caregivers, students had more resources to help them implement adaptations for cognitive impairments.

First of all, a way to encourage autonomy is to use a simplified interface. For example, the way to access the quiz to play can be simplified, e.g., the elder click on his or her picture to play instead of using a login/password. When menus or configurations are more complex, it is an excellent opportunity to involve caregivers instead of end-users to avoid frustrations. Simplifying the application also implies modi- 
What is this plant that evokes the Mexican desert?

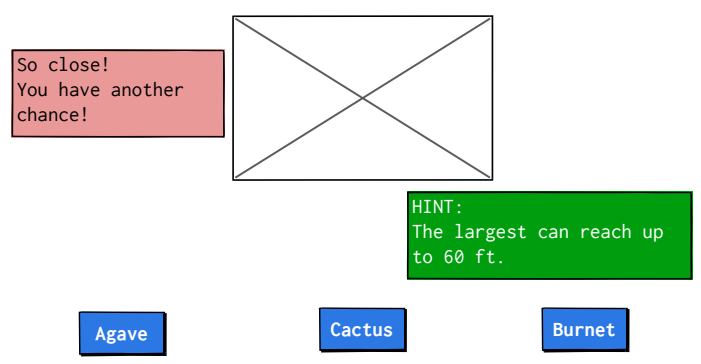

(a) Using hints to encourage the player

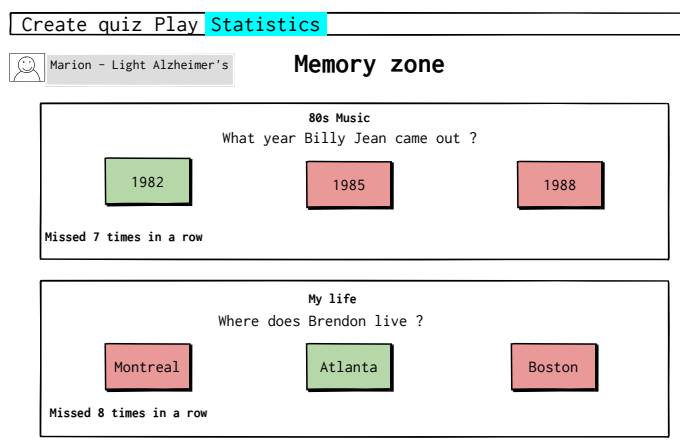

(b) Identifying which questions are always failed by a given user

Fig. 6: Cognitive adaptations

fying the business logic. For example, several applications offered to re-ask a failed question later during the game, with the wrong answer removed, helping elders memorizing the correct answer. Several teams also provided hints (Fig. 6a), or restricted the number of questions or available answers.

Another way to adjust to Alzheimer's levels is to present more or less information to the user. Too much information would confuse a user with advanced Alzheimer's and, at the same time, would reassure a patient at a lower stage. Finding a way to adapt the application to support a specific individual is challenging. That challenge can be tackled in different ways. One way to ensure that every older adult's requirements are met is by creating a personalized profile. The point here is to identify the link between the technical adaptation and the requirement that triggered it. Personality traits and dependency levels were also recorded in many cases.

Encouragement is a straightforward way to support elders who are not familiar with the technology. Based on users' feedback, the best ones are personalized and sound sincere (e.g., "Great performance Jean!" and "It is OK, Alexandra!"). Working on encouragement allowed students to experiment with good ideas that went bad in the end. For example, encouraging someone using sentences like "So close, you got 2 good answers out of 3!" emphasized the failure and was rejected by elders.
One of the best cognitive features was the definition of tailored quizzes: it is possible to create quizzes about events from their lives to maintain elders' memories alive. It also give the opportunity to adjust to older adults coming from different backgrounds. In other cases, the application allowed cloning a quiz and adapting it for different users, for example, by taking a shared quiz and removing hard questions for a participant. Adaptive quizzes with increasing difficulty can also be implemented that way. The point here is to prevent elders from feeling defeated for some memory they have lost for good, bringing up negative emotions such as anxiety and aggressiveness.

One team proposed an adaptation complementary to the precedent: a Memory Zone (Fig. 6b). With this adaptation, a caregiver can see all the systematically failed questions, helping the medical staff map the memory losses. This is an excellent example of how meaningful statistics are essential in this context and how the interaction from one stakeholder (the elder) can be monitored to ease the medical staff's job, ultimately helping the elder.

Taking a step back, cognitive impairment adaptations were more guided than the two other families. However, students demonstrated much creativity in collecting requirements, designing, and then implementing their proposition. They even came up with unforeseen adaptations (e.g., the Memory zone).

d) With Respect to multi-adaptations: Many teams tried to implements adaptation for more than one type of impairment. It usually consists of a very well-thought-out major adaptation and another hastily designed (for example, a bigger font). Students showed a willingness to implement such adaptation but were often unable to deliver it for time reasons.

\section{Lessons learned \& Perspectives}

First of all, information sharing between institutions is essential. For example, when UCA let students work on visual impairment without providing a simulation tool, UQAM demonstrated the tool during the lectures. It allowed us to observe that none of the solutions is better than the other, as they both introduce biases in the results. Another lesson learned thanks to the sharing of the course experience was related to motor impairments. As UQAM was developing mobile applications, it was easier to address these requirements thanks to the mobile device features (e.g., accelerometers).

On June 21, we created a questionnaire to gather feedback from students concerning this course in both institutions. We received 50 answers, where $60 \%$ were submitted by students who followed the course described in Sec. IV. The point here was not to implement a quantitative study but to gather feedback from students in an open way to feed the subsequent instances of this course.

First of all, only $4 \%$ of students were not interested in the ageing population, while $50 \%$ were very interested in this issue. Moreover, $66 \%$ of the respondents stated that this experience positively influenced their knowledge of elders' needs. More importantly, students expressed feedback such as "Exciting project because we are looking at societal issues 
that affect more and more people today and that we have to consider". They also pointed out that some of the disorders studied during the course may affect other people temporarily or permanently (e.g., "When you're lucky enough not to have a particular disorder, you don't necessarily realize the difficulties that this can cause daily").

From an RE perspective, students explicitly mentioned the importance of taking end-users into account in their development (30\%): "Do not code right away but make a model to present to end-users. Make personas / scenarios. Organize users' evaluations with potential users. Include users with disabilities in a project.". Combining this aspect with project management and development techniques was also highlighted (33\%): "Agile development is ideal to adapt our product along the way. Teamwork and feedback are essential to the success of a large project".

In terms of operational aspects (16\%), students mentioned that contact with end-users (caregivers or elders) have been more complex and going through intermediaries is not always the best solution: "It is better to have direct contact with users to understand their needs better". However, students also identified that working remotely helped them be more productive, as the formal logistics (timed appointments every week, instant messaging) helped them stay on track. They also mentioned that the choice of web technologies at the implementation level without a dedicated web programming course as a prerequisite slowed them down.

At the educational level, the difficulties encountered by the instructors are to combine technological achievements with societal achievements to obtain a usable and exciting result for elders. It is necessary to have a weekly follow-up to overcome the students' difficulties and provide feedback on the fly. Using personas and user stories to express requirements was the key to staying focused on user expectations and offering adapted solutions. Allowing the students to define their scope helps calibrate the results according to their technical skills and interests. Regularly validating progress concerning users and educational expectations guarantees that quality applications are obtained in the end.

We have noted that dealing with several disorders is too complex for the students in the available time. It seems that it would be more appropriate to give the students more precise requirements by explicitly specifying the expectations for the specified multiple impairments. However, the benefit of doing this is unclear, as the requirement collection phase is essential in the project to support learning.

The relationship with health partners is fundamental for the instructor team. It allowed us to acquire a better knowledge of the needs and expectations according to the impairments. It also allows students to work in a societal framework. It is essential to find the right level of exchange, which is difficult when the cohort of students is significant, particularly in the end-user evaluation phases. Having several partners involved depending on the disorders helps to distribute the loads. However, our vision might be biased by decades of collaboration with health practitioners. We were hoping to evaluate the course implementation at UQAM to check this assumption, but the COVID situation blocked all interaction with health practitioners during summer 2020.

\section{CONCLUSIONS}

Leveraging decades of collaboration with health professionals, we have created a course dedicated to developing software while considering specific requirements from elders. Our objective is to raise awareness of inclusive requirement engineering and software development to the next generation of software developers. The course material is available on a dedicated website ${ }^{8}$ for french-speaking institutions.

From a requirement engineering perspective, collecting requirements using personas and user stories makes it possible to stay as close as possible to users' needs. An agile project management approach, including regular user feedback, makes it possible not to drift and have several iterations directed by the prioritized needs of the targeted users. Moreover, the definition of the scope of the solution by the students allows the proposals to be calibrated according to their expertise and interests. Considering how ageing can impact users, a key lesson here is to take the customization into account in the way software is designed, as in the end we are all different.

Being aware of the ageing population and its specificity gave students openness for their future developments. For example, a former student who followed the course won the first place in the HACKCOVID19 competition $^{9}$ with a project dedicated to helping the elders to stay in contact with their loved ones without having to solicit the staff of their institution. Close cooperation upstream and during classes with associative or scientific partners in age-related health fields is also a significant asset for improving the teaching quality.

Considering the ageing population is essential and can be done in various contexts. Based on the expertise of the instructors' team, we focused on software engineering, humancomputer interfaces and artificial intelligence. However, the ageing population can be addressed with courses related to the internet of things, speech or image recognition. Nevertheless, in any case, all these domains heavily rely on requirements engineering to collect the needs from the elders and caregivers. We decided to focus on impairments to make the experience more explicit for students. However, it is also possible to focus on residence monitoring or mobility applications, for example. In this respect, the approach presented in this article can be used in other courses and other universities.

\section{ACKNOWLEDGMENTS}

The work reported in this paper is funded by the Conseil franco-québécois de coopération universitaire (Fond de Recherche du Québec - Nature et technologie, Samuel de Champlain program - 2020-FQ-278393). Authors want to thank Nicolas Ferry, Jean-Michel Bruel, Rémi Pourtier, Christian Brel, Prune Pilone, and Alison Lecuyer for their contribution to this work, as well as the staff from the Centre d'accueil

\footnotetext{
${ }^{8}$ https://ace-design.github.io/champlain/, in French

${ }^{9}$ https://www.hec.edu/fr/news-room/succes-du-hackathon-hacking-covid-19
} 
TABLE II: Adaptations implemented by students at UCA (Winter 2021)

The three following tables list all the adaptation designed by students at UCA during Winter 2021. The adaptations are assessed according to two dimensions: ( $i$ ) their added-value for the elders and (ii) their technical difficulty at the implementation level. The number between parenthesis represents the number of teams that implemented such adaptation in their product. When two numbers are present (e.g., 5/1), the second number represent the number of teams that planned to implement such adaptation but did not deliver in the end.

\begin{tabular}{c||ccc} 
Assessment & Low-value & Medium & High-value \\
\hline \hline Easy & Text color choice (1) & Background color choice (2) & Font size adjustment (9) \\
& Night mode (1) & Max. number of words per question (1) & Enlarged content (2) \\
& & Color coded for color blindness (2) & Bold buttons borders (2) \\
& & All Caps text (1) & Bold text (3) \\
Medium & - & Modify contrast/luminosity (5/1) & Confirmation after click (2) \\
& & Font choices (5) & Simplified interface (3) \\
Hard & - & Screen Reader (-/1) & Push content to sides (3)
\end{tabular}

(a) Visual Adaptations

\begin{tabular}{c||ccc} 
Assessment & Low-value & Medium & High-value \\
\hline \hline Easy & - & - & Enlarged Button (2) \\
Medium & - & - & Delay between two clicks (1) \\
Hard & - & Mouse stroke recording (1) & Vocal commands (1)
\end{tabular}

(b) Motor Adaptations

\begin{tabular}{c||ccc} 
Assessment & Low-value & Medium & High-value \\
\hline \hline Easy & Information on quiz (3) & Max. number of answer (2) & Encouragements (4) \\
& & Max. number of questions (3) & Second chance or more (3) \\
& & Skip a hard question (3) & Two or more chances (3) \\
& & Ask for confirmation (3) & Most forgotten questions (1) \\
& & Hide the score (3) & Hint on questions (3) \\
& Toggle random or fixed question order (1) & Quiz summary (4) & Personal Quiz (3) \\
& & Remove wrong answer (4) & Revisit failed questions (1) \\
Hard & & & mage as response (4) \\
& & & Meaningful statistics (4)
\end{tabular}

(c) Cognitive Adaptations

de jour de Biot who invested an invaluable amount of time in advising.

\section{REFERENCES}

[1] P. Demeny, G. McNicoll, and D. Hodgson, Encyclopedia of Population. Fairfield, CN: Sociology \& Anthropology Faculty Book and Media Gallery, Fairfield University, 2003.

[2] N. Bohnert, J. Chagnon, and P. Dion, "Population Projections for Canada (2013 to 2063), Provinces and Territories (2013 to 2038) - 91-520-X (archived)," Statistics Canada, Minister of Industry, Ottawa, ON, Canada, Tech. Rep. 978-1-100-25031-1, May 2015. [Online]. Available: https: //www150.statcan.gc.ca/n1/pub/91-520-x/91-520-x2014001-eng.pdf

[3] Statistics Canada, "Historical Age Pyramid," Ottawa, ON, Canada, 2016, accessed: 2021-06-15. [Online]. Available: https://www12.statcan.gc.ca/census-recensement/2016/dp-pd/ pyramid/pyramid.cfm?type $=1 \backslash \&$ geo $1=01$

[4] - "Population Projections for Canada (2018 to 2068), Provinces and Territories (2018 to 2043) - 91-520-X," Minister of Industry, Ottawa, ON, Canada, Tech. Rep. 978-0-660-31280-4, Sep. 2019. [Online]. Available: https://www150.statcan.gc.ca/n1/pub/ 91-520-x/91-520-x2019001-eng.htm

[5] AGE-WELL, Canada's Technology and Aging Network, "Annual Report: COVID-19, technology and the way forward," Networks of Centres of Excellence, Canada, Tech. Rep. 2019-2020, 2020. [Online]. Available: https://agewell-nce.ca/wp-content/uploads/2021/01/ AGEWELL_ENG_AR_2020.pdf

[6] A.-M. Déry-Pinna, A. Giboin, and P. Renevier-Gonin, "Design of Digital Therapeutic Workshops for People with Alzheimer's Disease," in Universal Access in Human-Computer Interaction. Design Methods and User Experience, Held as Part of the 23nd International Conference, HCII 2021, ser. Lecture Notes in Computer Science, vol. 12768. Springer, 2021, pp. 1-15. [Online]. Available: https://doi.org/10.1007/978-3-030-78092-0_24
[7] K. Lan and W. Shih, "Early diagnosis of parkinson's disease using a smartphone," in The 9th International Conference on Future Networks and Communications (FNC'14)/ The 11th International Conference on Mobile Systems and Pervasive Computing (MobiSPC'14) / Affiliated Workshops, August 17-20, 2014, Niagara Falls, Canada, ser. Procedia Computer Science, vol. 34. Elsevier, 2014, pp. 305-312. [Online]. Available: https://doi.org/10.1016/j.procs.2014.07.028

[8] Y. Vaizman, K. Ellis, and G. R. G. Lanckriet, "Recognizing detailed human context in-the-wild from smartphones and smartwatches," CoRR, vol. abs/1609.06354, 2016. [Online]. Available: http://arxiv.org/ abs/1609.06354

[9] H. Chi, E. Agama, and Z. G. Prodanoff, "Developing serious games to promote cognitive abilities for the elderly," in 5th IEEE International Conference on Serious Games and Applications for Health, SeGAH 2017, Perth, Australia, April 2-4, 2017, N. Dias, S. de Freitas, D. Duque, N. F. Rodrigues, K. Wong, and J. L. Vilaça, Eds. IEEE Computer Society, 2017, pp. 1-8. [Online]. Available: https://doi.org/10.1109/SeGAH.2017.7939279

[10] N. N. B. Abdullah, J. Grundy, J. McIntosh, Y. C. How, S. Saharuddin, K. K. Tat, E. ShinYe, A. J. A. Rastom, and N. L. Othman, "Using work system design, user stories and emotional goal modeling for an mhealth system," in 2020 IEEE First International Workshop on Requirements Engineering for Well-Being, Aging, and Health (REWBAH), 2020, pp. $1-10$.

[11] T. Sedano, P. Ralph, and C. Péraire, "The Product Backlog," in Proceedings of the 41st International Conference on Software Engineering, ICSE 2019, Montreal, QC, Canada, May 25-31, 2019, J. M. Atlee, T. Bultan, and J. Whittle, Eds. IEEE / ACM, 2019, pp. 200-211. [Online]. Available: https://doi.org/10.1109/ICSE.2019.00036

[12] J. Horkoff, "The Influence of Agile Methods on Requirements Engineering Courses," in 8th IEEE International Workshop on Requirements Engineering Education and Training, REET@RE 2018, Banff, AB, Canada, August 21, 2018, M. Moshirpour, M. Moussavi, A. M. Grubb, S. Gregory, and B. H. Far, Eds. IEEE Computer 
Society, 2018, pp. 11-19. [Online]. Available: https://doi.org/10.1109/ REET.2018.00007

[13] J.-Y. Mao, K. Vredenburg, P. W. Smith, and T. Carey, "The state of user-centered design practice," Communications of the ACM, vol. 48, no. 3, pp. 105-109, 2005.

[14] Web Content Accessibility Guidelines (WCAG) 2.1, web, Web Content Accessibility Guidelines Working Group Recommendation, Dec. 2018. [Online]. Available: http://www.w3.org/TR/WCAG20/ 